\title{
NEUTRON PRODUCTION IN BLACK HOLE CORONAE AND PROTON LOADING OF JETS*
}

\author{
GABRIELA S. VILA $^{\dagger, \S}$, FLORENCIA L. VIEYRO ${ }^{\dagger,} \boldsymbol{\Phi}^{\text {and }}$ GUSTAVO E. ROMERO ${ }^{\dagger, \ddagger}, \|$ \\ ${ }^{\dagger}$ Instituto Argentino de Radioastronomía, \\ C.C. 5, (1894) Villa Elisa, Buenos Aires, Argentina \\ ${ }^{\ddagger}$ Facultad de Cs. Astronómicas y Geofísicas, \\ Universidad Nacional de La Plata, Paseo del Bosque S/N, \\ (1900) La Plata, Buenos Aires, Argentina \\ §gvila@iar-conicet.gov.ar \\ \fvieyro@iar-conicet.gov.ar \\ "1romero@iar-conicet.gov.ar
}

Received 30 October 2013

Revised 6 December 2013

Published 21 March 2014

\begin{abstract}
We study the production of neutrons in the corona of an accreting black hole through the interaction of locally accelerated protons with matter and radiation. A fraction of these neutrons may escape and penetrate into the base of the jet, later decaying into protons. This is a possible mechanism for loading Poynting-dominated outflows with baryons.

We characterize the spatial and energy distribution of neutrons in the corona and that of the protons injected in the jet by neutron decay. We assess the contribution of these protons to the radiative spectrum of the jet. We also investigate the fate of the neutrons that escape the corona into the external medium.
\end{abstract}

Keywords: X-ray binaries; radiation mechanisms: non-thermal; astrophysical jets.

PACS Numbers: 97.80.Jp, 97.10.Gz, 95.30.Cq

\section{Introduction}

The composition of relativistic outflows is presently uncertain. Radio synchrotron emission positively discloses the presence of (non-thermal) electrons/positrons in relativistic jets. Only for two system (the microquasars SS 433 and 4U1630-47, see Refs. 1 and 2) there exists direct evidence that the jets contain baryons - namely iron nuclei - too. Jets with a high content of baryons should carry a large amount of kinetic energy. The baryonic component of jets, however, may remain "dark" and thus undetected if the baryons cannot be accelerated or do not find suitable targets to interact and radiate efficiently.

${ }^{*}$ This is an Open Access article published by World Scientific Publishing Company. It is distributed under the terms of the Creative Commons Attribution 3.0 (CC-BY) License. Further distribution of this work is permitted, provided the original work is properly cited. 
The problem of baryon loading of relativistic jets is related both to the origin of the jets and their interaction with the external medium. It is expected that diskdriven outflows contain protons and heavier nuclei since they are fed directly from the accretion flow. Jets powered by the rotation of a black hole start basically as an electromagnetic flux but may get loaded with baryons by entrainment, for example with matter from a slow outflow launched by the accretion disk or from the wind of a companion star. ${ }^{3}$

Both in low-luminosity active galactic nuclei (AGN) and in X-ray binaries (XRBs), observations support the existence of a very hot, advection-dominated and radiatively inefficient cloud of plasma around the compact object. In XRBs this "corona" is postulated to be the emission site of the power-law hard X-ray spectrum observed during the low-hard state. The corona has been classically modeled as a two-temperature ion-electron thermal plasma, although some models include also non-thermal electrons or electron-positron pairs, see e.g. Refs. 4-6. More recently, Refs. 7-9 have investigated the consequences of the injection of a population of relativistic protons in the corona of black holes in XRBs. The emission in the MeV band predicted by this model can reproduce very well that detected from Cygnus $\mathrm{X}-1$ by COMPTEL and INTEGRAL. ${ }^{12-14}$

The relativistic protons in the corona interact with thermal protons and with radiation to produce pions. In some collisions the proton is converted into a neutron. These energetic neutrons leave the source basically unaffected by energy losses and propagate for long distances before decaying into a proton, an electron and a neutrino. The production and escape of neutrons in AGN has been studied before by several authors, see e.g. Refs. 15-19. If a pair of jets is launched from the vicinities of the black hole, a fraction of the neutrons will decay inside them. The charged products of the decay will get trapped by the magnetic field and will be advected with the outflows, thus loading them with protons (and electrons) with a non-thermal energy distribution.

In this work we study the production of neutrons by hadronic interactions in the magnetized corona of a XRB with jets (a microquasar). We calculate the spectrum of neutrons inside the corona in steady state and show that they can escape almost freely. We then compute the distribution in energy and space of neutrons outside the corona, and those of the protons and electrons at the point where they are created. We estimate the high-energy emission from the protons injected inside the jets in the case they interact with a dense clump in the wind of the companion star. Finally, we discuss the characteristics of the radio emission expected from the electrons injected far from the corona.

\section{Basics of the Model and Results}

The corona model we adopt is described in detail in Refs. 7-9. We consider a spherical and homogeneous corona around a black hole of $15 M_{\odot}$ (similar to that in Cygnus $\mathrm{X}-1$ ). The radius of the hot cloud is $R_{\mathrm{c}}=35 R_{\text {grav }}$ and its bolometric 
luminosity $L_{\mathrm{c}}=0.01 L_{\mathrm{Edd}} \approx 10^{37} \mathrm{erg} \mathrm{s}^{-1}$. The mean number density of thermal protons and electrons in the corona is $n_{p, e} \approx 10^{13} \mathrm{~cm}^{-3}$ and the mean magnetic field strength $B_{\mathrm{c}} \approx 10^{5} \mathrm{G}$. Relativistic protons are accelerated in the corona by fast magnetic reconnection. At injection the energy spectrum of relativistic protons is a power-law $Q_{p} \propto E_{p}^{-\alpha}$ (in units of $\mathrm{erg}^{-1} \mathrm{~cm}^{-3} \mathrm{~s}^{-1}$ ) of index $\alpha=2.2$. $^{\mathrm{a}}$ We calculate the proton distribution $N_{p}\left(E_{p}\right)$ (in units of $\mathrm{erg}^{-1} \mathrm{~cm}^{-3}$ ) in steady state solving a set of coupled transport equations that include energy losses, escape and injection.

The relativistic protons interact with the thermal protons, the photon field from the accretion disk (that we model as a blackbody), the non-thermal radiation produced by the relativistic particles, and the Comptonization radiation field of the corona. The latter is the most important; we parametrize it as $n_{\mathrm{ph}} \propto$ $E_{\mathrm{ph}}^{-\beta} \exp \left(-E_{\mathrm{ph}} / E_{0}\right)$ with $\beta=1.6$ and $E_{0}=150 \mathrm{keV}$. Proton-proton $(p p)$ and proton-photon $(p \gamma)$ collisions produce neutrons through the reactions

$$
\begin{aligned}
& p+p \rightarrow p+n+\pi^{+}+a \pi^{0}+b\left(\pi^{+}+\pi\right), \\
& p+p \rightarrow n+n+2 \pi^{+}+a \pi^{0}+b\left(\pi^{+}+\pi\right), \\
& p+\gamma \rightarrow n+\pi^{+}+a \pi^{0}+b\left(\pi^{+}+\pi\right),
\end{aligned}
$$

where $a, b$ are integers. For the neutron injection functions we use the parametrizations given in Refs. 15-18; the results are plotted in the left panel of Fig. 1. The total power injected in neutrons inside the corona is $L_{n} \approx 6 \times 10^{35} \mathrm{erg} \mathrm{s}^{-1}$. The right panel of Fig. 1 shows the cooling rates for neutrons by collisions with protons and photons, the decay rate, and the inverse of the corona crossing time. The escape time is the shortest for all energies considered, so we assume that neutrons leave the corona without losing energy.
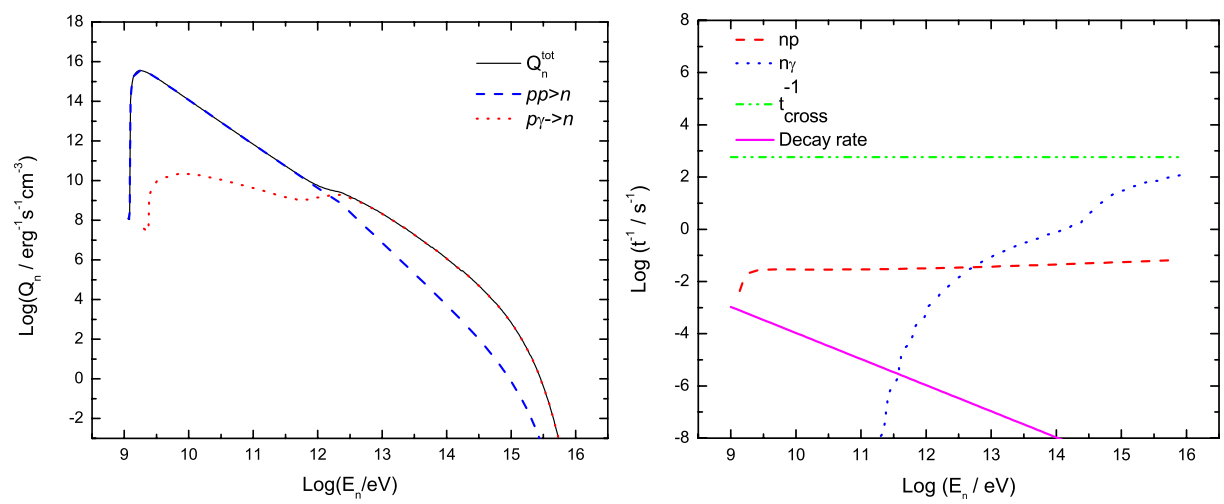

Fig. 1. Left: neutron injection function inside the corona. Right: neutron cooling, escape, and decay rates in the corona.

a The allowed range of values of the spectral index of particles accelerated via a Fermi I-type mechanism at magnetic reconnection sites in an open issue; see e.g. Refs. 10 and 11. 

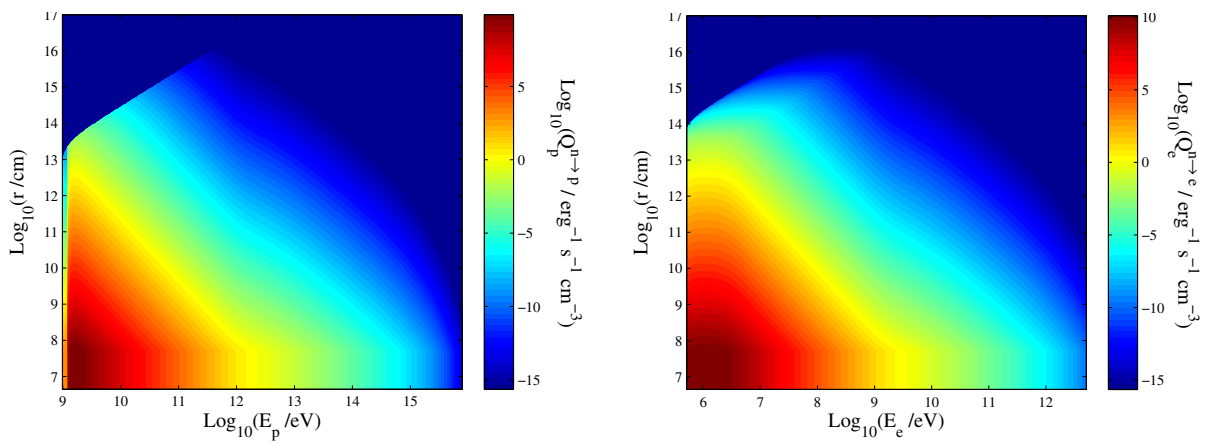

Fig. 2. Left: spectrum of protons injected by decay of neutrons as a function of energy and distance to the black hole. Right: the same but for electrons.

Outside the corona the steady-state distribution of neutrons in energy and space is $N_{n}\left(E_{n}, r\right) \propto r^{-2} \exp \left(-r / r_{\tau}\right)$, where $r_{\tau}=\tau_{n} v_{n}$, and $\tau\left(E_{n}\right)$ and $v_{n}\left(E_{n}\right)$ are the mean lifetime and the velocity field of the neutrons, respectively. When decaying each neutron injects a proton, an electron and a neutrino, $n \rightarrow p+e^{-}+\bar{\nu}_{e}$. The proton takes $\sim 99.9 \%$ of the energy of the neutron, so we can approximate the proton injection function as $Q_{p}^{n \rightarrow p}\left(E_{p}, r\right) \approx N_{n}\left(E_{n}, r\right) / \tau\left(E_{n}\right)$ with $E_{p}=0.999 E_{n}$. We calculated the injection rate of electrons $Q_{e}^{n \rightarrow e}$ as in Ref. 21. Figure 2 shows the distribution in energy and space of protons and electrons. The total power injected in protons is $\gtrsim 10^{35} \mathrm{erg} \mathrm{s}^{-1}$ whereas the power in electrons is $\sim 10^{30} \mathrm{erg} \mathrm{s}^{-1}$. Most of these particles are created in a region of the size of the binary system, $r \lesssim 10^{12-13} \mathrm{~cm}$.

A fraction of the neutrons will decay inside the jet loading it with charged particles; here we will be interested in the fate of the protons. To characterize the jet we applied the model developed in Refs. 23 and 24. Two conical jets are launched at a distance of $50 R_{\text {grav }}$ from the black hole with a half opening angle $\sim 6^{\circ}$. For this geometry, the decay of neutrons along the jets injects a total power $\sim 10^{34}$ $\operatorname{erg~} \mathrm{s}^{-1}$ in protons. The protons may interact with a clump from the wind of a massive companion star that penetrates the jet, producing gamma rays by neutral pion decays from $p p$ collisions. ${ }^{25}$ We estimate the gamma-ray spectrum for different combinations of the size and density of the clump and its position along the jet. The results are shown in Fig. 3.

Electrons injected within the binary system interact with the magnetic field and the radiation field of the companion star. For typical values of a massive star, $B_{\star} \sim 100 \mathrm{G}$ and $T_{*} \sim 10^{4} \mathrm{~K}$, the cooling times are very long and the electrons diffuse far from the site where they were created. A detailed calculation of the radiative spectrum of the electrons and of the morphology of the emission region must be carried out accounting for propagation effects as done in Ref. 22. As a zeroth-order estimate, however, we expect the formation of a "nebula" of radio synchrotron emission at $\sim \mathrm{GHz}$ frequencies. If most of the power injected in electrons 


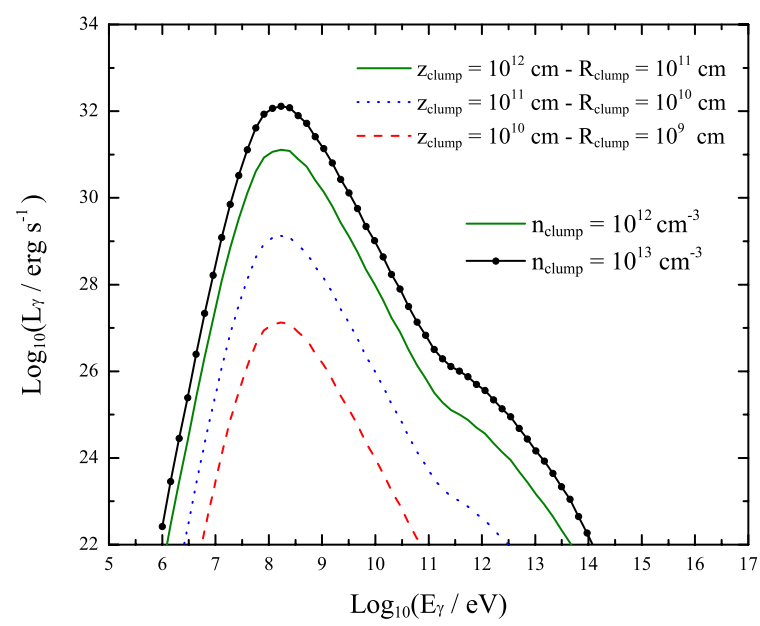

Fig. 3. Gamma-ray emission from $p p$ collisions in jet-clump interactions, for different values of the clump radius $R_{\text {clump }}$, density $n_{\text {clump }}$, and distance to the black hole $z_{\text {clump }}$.

were radiated through this channel, the nebula would be detectable at the level of the mJy at $1 \mathrm{GHz}$ for a source located at $\sim 2 \mathrm{kpc}$.

\section{Discussion and Perspectives}

We have studied the production of neutrons in the corona around an accreting black hole and the injection of protons and electrons from neutron decay outside the source. In a system with jets, this is a way of loading the outflows with baryons independently of the launching mechanism. Although we have applied the model to XRBs, it is straightforward to extend it to AGN. Protons injected in the jets will produce gamma rays if they can interact with a dense target such as a clump from the wind of the companion star. Although the expected hadronic luminosity is only $\lesssim 10^{32} \mathrm{erg} \mathrm{s}^{-1}$, the added contribution of several of these events may give rise to weak flaring emission at $\mathrm{GeV}$ energies, as has been observed in Cygnus X$1 .^{26}$ The interaction of these protons with other targets, such as the stellar wind or a molecular cloud, remains to be investigated. The cooling of electrons injected outside the corona may create a detectable radio nebula at scales of the size of the binary system. The detailed structure and emissivity of the nebula will be addressed in a forthcoming work.

\section{Acknowledgments}

G.S.V. wishes to thank the High-Energy Astrophysics Theory Group of the MaxPlanck-Institut für Kernphysik for their hospitality during her stay and the preparation of this work. This research was supported by grants PIP 0078/2010 and PICT 2012-00878 Préstamo BID. 


\section{References}

1. S. Migliari, R. Fender and M. Méndez, Science 297, 1673 (2002).

2. M. Díaz-Trigo, J. C. A. Miller-Jones, S. Migliari, J. W. Broderick and T. Tzioumis, Nature, in press, published online first (2013).

3. G. E. Romero, D. F. Torres, M. M. Kaufman Bernadó and I. F. Mirabel, A $\& A 4$ 410, L1 (2003).

4. J. Poutanen, Accretion disc-corona models and $\mathrm{X} / \gamma$-ray spectra of accreting black holes, in Theory of Black Hole Accretion Disks, eds. M. A. Abramowicz, G. Bjornsson and J. E. Pringle (Cambridge University Press, Cambridge 1998), p. 100.

5. R. Belmont, J. Malzac and A. Marcowith, A\&SA 491, 617 (2008).

6. I. Vurm and J. Poutanen, ApJ 698, 29 (2009).

7. F. L. Vieyro, Y. Sestayo, G. E. Romero and J. M. Paredes, A\&A 546, id.A46 (2012).

8. F. L. Vieyro and G. E. Romero, A\&A 542, id.A7 (2012).

9. G. E. Romero, F. L. Vieyro and G. S. Vila, $A \mathscr{G} A$ 519, id.A109 (2010).

10. E. M. de Gouveia dal Pino and A. Lazarian, $A \& A$ 441, 845 (2005).

11. L. O'C. Drury, MNRAS 422, 2474 (2012).

12. M. L. McConnell, J. M. Ryan, W. Collmar et al., ApJ 543, 928 (2000).

13. M. Cadolle Bel, P. Sizun, A. Goldwurm et al., A\&SA 446, 591 (2006).

14. E. Jourdain, J. P. Roques and J. Malzac, ApJ 744, id.64 (2012).

15. M. Sikora, M. Begelman and B. Rudak, ApJ 341, L33 (1989).

16. A. Atoyan, $A \mathscr{E} A$ 257, 465 (1992).

17. W. Bednarek, A\&SA 264, 331 (1992).

18. A. M. Atoyan and C. D. Dermer, ApJ 586, 79 (2003).

19. K. Toma and F. Takahara, ApJ 754, 148 (2012).

20. M. Begelman, B. Rudak and M. Sikora, ApJ 362, 38 (1990).

21. P. B. Abraham, K. A. Brunstein and T. L. Cline, Phys. Rev. 150, 1088 (1966).

22. V. Bosch-Ramon and D. Khangulyan, PASJ 63, 1023 (2011).

23. G. E. Romero and G. S. Vila, $A \& A$ 485, 623 (2008).

24. G. S. Vila, G. E. Romero and N. A. Casco, $A \& A$ 538, id.A97 (2012).

25. A. T. Araudo, V. Bosch-Ramon and G. E. Romero, A $\& A$ 503, 673 (2009).

26. A. Bodaghee et al., ApJ 775, id.98 (2013). 